\title{
Unified view of correlations using the square-norm distance
}

\author{
Bruno Bellomo, ${ }^{1}$ Gian Luca Giorgi, ${ }^{2}$ Fernando Galve, ${ }^{2}$ Rosario Lo Franco, ${ }^{1,3}$ Giuseppe Compagno, ${ }^{1}$ and Roberta Zambrini ${ }^{2}$ \\ ${ }^{1}$ Dipartimento di Fisica, Università di Palermo, via Archirafi 36, I-90123 Palermo, Italy \\ ${ }^{2}$ IFISC (UIB-CSIC), Instituto de Física Interdisciplinar y Sistemas Complejos, UIB Campus, E-07122 Palma de Mallorca, Spain \\ ${ }^{3}$ CSFNSM and Dipartimento di Fisica e Astronomia, Università di Catania, Viale A. Doria 6, I-95125 Catania, Italy
}

(Received 29 December 2011; published 5 March 2012)

\begin{abstract}
The distance between a quantum state and its closest state not having a certain property has been used to quantify the amount of correlations corresponding to that property. This approach allows a unified view of the various kinds of correlations present in a quantum system. In particular, using relative entropy as a distance measure, total correlations can be meaningfully separated into a quantum part and a classical part thanks to an additive relation involving only the distances between states. Here we investigate a unified view of correlations using as a distance measure the square norm, which has already been used to define the so-called geometric quantum discord. We thus also consider geometric quantifiers for total and classical correlations, finding, for a quite general class of bipartite states, their explicit expressions. We analyze the relationship among geometric total, quantum, and classical correlations, and we find that they no longer satisfy a closed additivity relation.
\end{abstract}

DOI: 10.1103/PhysRevA.85.032104

PACS number(s): 03.65.Ud, 03.67.Mn, 03.65.Yz

\section{INTRODUCTION}

Quantum systems have properties characterized by various kinds of correlations, some of which distinguish them from classical systems [1,2]. These properties may be an essential resource for quantum computation and quantum information [3]. This makes it important to distinguish those correlations that are particular to quantum systems from the ones present also in classical systems. A nonlocal property of quantum systems, entanglement, allows us to achieve exponential speedup in pure-state computation if it grows with the size of the system [4]. On the other hand, in the case of mixed-state computation, in certain computational tasks quantum speedup can be achieved using separable (unentangled) states, such as in the so-called deterministic quantum computation with one qubit (DQC1) protocol [5]. This speedup has been linked [6] to the presence of quantum discord [7,8], considered to be a quantifier of the quantum part of correlations present in a bipartite system and defined as the difference between two quantum analogues of classical mutual information $[9,10]$.

In the general case of a multipartite system, the various kinds of correlations present in a quantum state have been linked to the distance between the system state itself and its closest states without the desired property, allowing us to look at them in a unified view [11,12]. Relative entropy, although not symmetrical under the exchange of the entries, has been used as a distance measure between states. In this approach, the decomposition of the total correlations $T$ in a classical part $C$ and a quantum part $D$ appears meaningful because $T$ equals the sum of $D$ and $C$ up to a quantity $L$, which in turn is a relative entropy-based (REB) distance between two of the relevant closest states. The quantity $L$ results to be, in the bipartite case, equal to the difference between REB quantum discord and its original definition $[7,8]$. Using relative entropy as a distance measure, correlation quantifiers therefore satisfy a closed additivity relation among them [12].

The properties of quantum discord have been widely investigated in recent years [13]. It has been shown that it is present in almost all quantum states [14], and the relation between discord and entanglement has been discussed
[15-17]. In contrast to entanglement, discord can be generated using local noise [18], and it is not monogamous [19]. Different from what happens for entanglement, quantum discord does not present sudden death [20] during its evolution but can still present revivals even in the absence of systemenvironment back action [21]. Generalizations of discord in the multipartite case have been also reported following different approaches [22].

On the other hand, both the original and the REB discord require involved minimization procedures even if only von Neumann (orthogonal) measurements are used. Using more general measurements [positive operator-valued measure (POVM)], the minimization problem becomes increasingly demanding [23], with the consequence that there are only a few general results. Discord analytical expressions have been obtained only for certain classes of two-qubit states, such as Bell diagonal [24], rank-2 [23,25], and $X$ [26] states and, for the case of continuous variables, for Gaussian states [27]. To overcome this drawback, geometric quantum discord $D_{\mathrm{g}}$ has been introduced based on the square-norm (Hilbert-Schmidt) distance between the system state and its closest classical state, and it has been used to evaluate quantum correlations present in an arbitrary two-qubit state [28]. Quantitative comparisons between REB and geometric discord have been reported [29-31], and their dynamics have been also compared, revealing qualitative differences in their time behaviors [32]. Geometric measures of total correlations, $T_{\mathrm{g}}$, and classical correlations, $C_{\mathrm{g}}$, have been also defined using the square-norm distance, with their explicit expressions given only for Bell diagonal states [32].

The aim of this paper is to discuss the role and use of the square-norm distance to quantify in a unified view various kinds of correlations in a two-qubit state. To this purpose we will consider a quite general class of bipartite states for which we will find explicit expressions for geometric quantifiers of total, quantum, and classical correlations. In analogy to what has been done with REB correlation quantifiers, we will investigate the possibility of having closed additive relations among correlation quantifiers based on the square norm. 
The main point of this paper is to show that relevant qualitative differences are found when one attempts to construct a unified view of correlations using different ways to measure the distance between the relevant states. In the case of a quite general class of two-qubit states ( $X$ states), we will be able to analytically prove that, different from what happens with REB distance, using the square-norm distance measure total correlations cannot be, in general, separated into a quantum part and a classical part satisfying an additive relation that involves only distances between states.

This paper is organized as follows: in Sec. II we introduce the framework of the unified view of correlations for both REB and its geometric counterpart. In Section III we present the class of states on which we will base our study, $X$ states; in Secs. IV and V we obtain the pertinent closest states. Finally, in Section VI we show under which conditions the closure of correlations is not satisfied and study how often and to what extent this happens for $X$ states.

\section{CORRELATIONS IN A QUANTUM STATE}

A natural and powerful way to quantify a given property of a quantum state consists of exploiting the distance between the state itself and its closest state without that property. Therefore, in this approach, it is necessary to choose a suitable distance measure. In this section, we first briefly review the correlation quantifiers defined by using relative entropy as a measure of distance between states, and we then describe the geometric correlation quantifiers based on the square-norm distance measure.

\section{A. Correlation quantifiers based on relative entropy}

Given two arbitrary multipartite states $\rho, \sigma$, their relative entropy is defined as $S(\rho \| \sigma)=-\operatorname{Tr}\left(\rho \log _{2} \sigma\right)-S(\rho)$, where $S(\rho)=-\operatorname{Tr}\left(\rho \log _{2} \rho\right)$ is the von Neumann entropy. Using relative entropy to quantify distances, the total correlations $T$ of state $\rho$ are defined by the distance between $\rho$ and the closest product state $\pi_{\rho}=\sigma_{A} \otimes \sigma_{B}$ (with $\sigma_{A(B)}$ being density matrices for the subsystems), $T=S\left(\rho \| \pi_{\rho}\right)$; discord (quantum correlations) $D$ is the distance between $\rho$ and the closest classical state $\chi_{\rho}=\sum_{i, j} p_{i, j}|i\rangle\langle i|\otimes| j\rangle\langle j|$ (with $\{|i\rangle,|j\rangle\}$ being independent local bases and $p_{i, j}$ being probabilities), $D=$ $S\left(\rho \| \chi_{\rho}\right)$, while classical correlations are the distance between $\chi_{\rho}$ and its closest product state $\pi_{\chi_{\rho}}, C=S\left(\chi_{\rho} \| \pi_{\chi_{\rho}}\right)$ [12]. We refer to these correlation quantifiers as REB quantifiers. In general, $T-(D+C) \neq 0$, but this difference is always equal to another quantity $L$ defined in terms of relative entropy, that is,

$$
T-D-C=-L,
$$

where $L=S\left(\pi_{\rho} \| \pi_{\chi_{\rho}}\right)$. The validity of Eq. (1) is proved by the fact that all the REB correlation quantifiers involved can be written as differences between von Neumann entropies as [12]

$$
\begin{aligned}
& T(\rho) \equiv S\left(\rho \| \pi_{\rho}\right)=S\left(\pi_{\rho}\right)-S(\rho), \\
& D(\rho) \equiv S\left(\rho \| \chi_{\rho}\right)=S\left(\chi_{\rho}\right)-S(\rho), \\
& C(\rho) \equiv S\left(\chi_{\rho} \| \pi_{\chi_{\rho}}\right)=S\left(\pi_{\chi_{\rho}}\right)-S\left(\chi_{\rho}\right), \\
& L(\rho) \equiv S\left(\pi_{\rho} \| \pi_{\chi_{\rho}}\right)=S\left(\pi_{\chi_{\rho}}\right)-S\left(\pi_{\rho}\right) .
\end{aligned}
$$

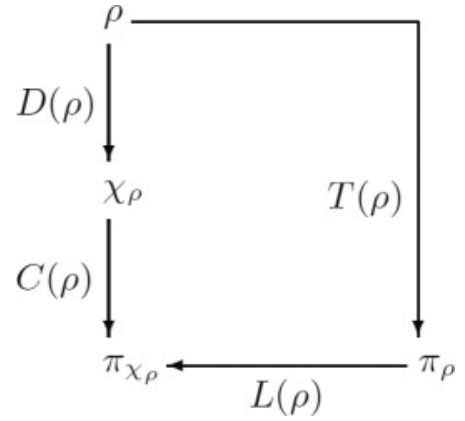

FIG. 1. Picture of the REB correlation quantifiers and the relevant states (see Ref. [12]).

These relations allow one to draw a simple diagram, proposed in Ref. [12] and displayed in Fig. 1, where each line refers to a kind of correlation and where the direction of each arrow is linked to the asymmetric definition of the relative entropy. For bipartite systems, the quantity $L$ exactly quantifies the difference between the REB discord $D$ of Eq. (2) and the original definition $\delta$ [7,8]: $\delta=D-L$. A closed additivity relation, Eq. (1), thus always holds among the REB correlation quantifiers.

\section{B. Correlation quantifiers based on the square norm}

As already noted, the REB correlation quantifiers have the drawback that their analytical expressions are known only for certain classes of states [24-27] and require, in general, numerical minimizations. A more manageable quantifier, called geometric quantum discord, has been recently introduced for quantum correlations as the square-norm distance between the system state $\rho$ and its closest classical state $\chi_{\rho}$ [28],

$$
D_{\mathrm{g}}(\rho)=\left\|\rho-\chi_{\rho}\right\|^{2},
$$

where $\|\cdot\|^{2}=\operatorname{Tr}(\cdot)^{2}$ is the square-norm distance in the Hilbert-Schmidt space and $\chi_{\rho}$ has the form $\chi_{\rho}=$ $\sum_{i, j} p_{i, j}|i\rangle\langle i| \otimes \rho_{j}^{B}$ (with $\{|i\rangle\}$ being a local basis on part $A, \rho_{j}^{B}$ being arbitrary states of part $B$, and $p_{i, j}$ being probabilities). The advantage of this definition is that $D_{\mathrm{g}}(\rho)$ can be analytically evaluated for an arbitrary two-qubit state and for some multipartite or higher-dimensional systems more easily than REB quantum discord [28,33]. It is worth noticing that the geometric definition of quantum discord of Eq. (3) is equal to the one obtained in analogy to the original definition of quantum discord and using the square-norm distance measure, that is, $D_{\mathrm{g}}(\rho)=\min _{\Pi^{A}}\left\|\rho-\Pi^{A}(\rho)\right\|^{2}$, where $\Pi^{A}(\rho)$ is the classical state resulting after a von Neumann measurement on part $A$ [33].

Because geometric discord is useful for quantifying quantum correlations in a system, it seems to be a natural extension to use the square norm also to define quantifiers of total and classical correlations as [32]

$$
T_{\mathrm{g}}(\rho) \equiv\left\|\rho-\pi_{\rho}\right\|^{2}, \quad C_{\mathrm{g}}(\rho) \equiv\left\|\chi_{\rho}-\pi_{\chi_{\rho}}\right\|^{2},
$$

where $\pi_{\rho}$ and $\pi_{\chi_{\rho}}$ are, respectively, the product states closest to $\rho$ and $\chi_{\rho}$ within the square-norm distance measure. We refer to the quantifiers based on square-norm distance as geometric 
correlation quantifiers. One can further define the quantity

$$
L_{\mathrm{g}}(\rho) \equiv\left\|\pi_{\rho}-\pi_{\chi_{\rho}}\right\|^{2}
$$

as the analog of the REB quantity $L$ in Eq. (2).

In analogy to what happens for REB discord, $D_{\mathrm{g}}$ can be written as a difference of purities, $D_{\mathrm{g}}=\operatorname{Tr}\left(\rho-\chi_{\rho}\right)^{2}=$ $\operatorname{Tr} \rho^{2}-\operatorname{Tr} \chi_{\rho}^{2}$. In fact, analyzing the results of Ref. [28], one can show that $\operatorname{Tr}\left(\rho \chi_{\rho}\right)=\operatorname{Tr}\left(\chi_{\rho}^{2}\right)$. However, as we shall see in Sec. VI, the other geometric correlation quantifiers $T_{\mathrm{g}}$ and $C_{\mathrm{g}}$ do not hold this property.

We shall now study the relationship among geometric correlation quantifiers by first finding their explicit expressions for a quite general class of bipartite states. We will also investigate if, in general, an additivity relation analogous to that of Eq. (1) is satisfied when geometric correlation quantifiers are used.

\section{TWO-QUBIT $X$ STATES}

In this section we describe the class of two-qubit states that we are going to use in our analysis. For our purposes it is useful to represent the states in the Bloch representation, which, for an arbitrary two-qubit state, is

$$
\begin{aligned}
\rho= & \frac{1}{4}\left[\mathbb{1} \otimes \mathbb{1}+\sum_{i} x_{i} \sigma_{i} \otimes \mathbb{1}\right. \\
& \left.+\sum_{i} y_{i} \otimes \mathbb{1} \sigma_{i}+\sum_{i, j} T_{i j} \sigma_{i} \otimes \sigma_{j}\right],
\end{aligned}
$$

where $\mathbb{1}$ is the $2 \times 2$ identity matrix, $\sigma_{i, j}(i, j=1,2,3)$ are the three Pauli matrices, $x_{i}=\operatorname{Tr}\left[\rho\left(\sigma_{i} \otimes \mathbb{1}\right)\right]$ and $y_{i}=\operatorname{Tr}[\rho(\mathbb{1} \otimes$ $\left.\left.\sigma_{i}\right)\right]$ are components of the local Bloch vectors $\vec{x}=\left\{x_{1}, x_{2}, x_{3}\right\}$ and $\vec{y}=\left\{y_{1}, y_{2}, y_{3}\right\}$, and $T_{i j}=\operatorname{Tr}\left[\rho\left(\sigma_{i} \otimes \sigma_{j}\right)\right]$ are components of the correlation tensor $T$.

In particular, we put our attention to the class of $X$ states, which are those states having nonzero elements only along the main diagonal and antidiagonal of the density matrix. The general structure of an $X$ density matrix is thus, in the standard basis $\mathcal{B}=\{|1\rangle \equiv|11\rangle,|2\rangle \equiv|10\rangle,|3\rangle \equiv|01\rangle,|4\rangle \equiv|00\rangle\}$,

$$
\rho_{X}=\left(\begin{array}{cccc}
\rho_{11} & 0 & 0 & \rho_{14} e^{i \gamma_{14}} \\
0 & \rho_{22} & \rho_{23} e^{i \gamma_{23}} & 0 \\
0 & \rho_{23} e^{-i \gamma_{23}} & \rho_{33} & 0 \\
\rho_{14} e^{-i \gamma_{14}} & 0 & 0 & \rho_{44}
\end{array}\right),
$$

where $\rho_{i j}(i, j=1,2,3,4)$ and $\gamma_{i, j}$ are all real, positive numbers. Bell states, Werner states, and Bell diagonal states belong to this class of states [34]. $X$-structure density matrices may arise in a wide variety of physical situations and are also experimentally achievable [35]. For example, $X$ states are encountered as eigenstates in all the systems with odd-even symmetry, such as in the Ising and the $X Y$ models [36]. Moreover, in many physical evolutions of open quantum systems an initial $X$ structure is maintained in time [37]. The parameters of the Bloch representation of Eq. (6) for an $X$ state are thus expressed in terms of the the density-matrix elements of Eq. (7) as [38]

$$
\begin{aligned}
& x_{3}=\rho_{11}+\rho_{22}-\rho_{33}-\rho_{44}, \\
& y_{3}=\rho_{11}-\rho_{22}+\rho_{33}-\rho_{44},
\end{aligned}
$$

$$
\begin{aligned}
& T_{11}=2 \cos \left(\gamma_{14}\right) \rho_{14}+2 \cos \left(\gamma_{23}\right) \rho_{23}, \\
& T_{12}=-2 \sin \left(\gamma_{14}\right) \rho_{14}+2 \sin \left(\gamma_{23}\right) \rho_{23}, \\
& T_{21}=-2 \sin \left(\gamma_{14}\right) \rho_{14}-2 \sin \left(\gamma_{23}\right) \rho_{23}, \\
& T_{22}=-2 \cos \left(\gamma_{14}\right) \rho_{14}+2 \cos \left(\gamma_{23}\right) \rho_{23}, \\
& T_{33}=\rho_{11}-\rho_{22}-\rho_{33}+\rho_{44},
\end{aligned}
$$

with $x_{1}=x_{2}=y_{1}=y_{2}=T_{13}=T_{23}=T_{31}=T_{32}=0$.

We are interested in the explicit expressions of the geometric correlation quantifiers for an $X$ state. In order to obtain them, we first need to find the relevant closest states when the distance is measured by the square norm.

\section{CLOSEST PRODUCT STATE}

In this section we are interested in finding the product state closest to a two-qubit $X$ state in the square-norm distance measure, with the aim being to obtain the geometric quantifier of total correlations. Indicating with $\rho_{A}=\frac{1}{2}\left[\mathbb{1}+\sum_{i} a_{i} \sigma_{i}\right]$ and $\rho_{B}=\frac{1}{2}\left[\mathbb{1}+\sum_{i} b_{i} \sigma_{i}\right]$ generic single-qubit states with Bloch vectors, respectively, $\vec{a}=\left\{a_{1}, a_{2}, a_{3}\right\}$ and $\vec{b}=\left\{b_{1}, b_{2}, b_{3}\right\}$, an arbitrary product state $\pi$ is given by their tensor product as

$$
\begin{aligned}
\pi=\rho_{A} \otimes \rho_{B}= & \frac{1}{4}\left[\mathbb{1} \otimes \mathbb{1}+\sum_{i} a_{i} \sigma_{i} \otimes \mathbb{1}+\sum_{i} b_{i} \mathbb{1} \otimes \sigma_{j}\right. \\
& \left.+\sum_{i, j} a_{i} b_{j} \sigma_{i} \otimes \sigma_{j}\right]
\end{aligned}
$$

The distance $F$ between an arbitrary two-qubit state $\rho$ as given in Eq. (6) and the product state $\pi$ using the square norm is then

$$
\begin{aligned}
F=\operatorname{Tr}(\rho-\pi)^{2}= & \frac{1}{4}\left[\sum_{i}\left(x_{i}-a_{i}\right)^{2}+\sum_{i}\left(y_{i}-b_{i}\right)^{2}\right. \\
& \left.+\sum_{i, j}\left(T_{i j}-a_{i} b_{j}\right)^{2}\right]
\end{aligned}
$$

The explicit form of the product state $\pi_{\rho}$ closest to $\rho$ is determined by the values of the variables $a_{i}, b_{i}$ as functions of the known state parameters $x_{i}, y_{i}, T_{i j}$, giving the absolute minimum of the distance $F$. Deriving $F$ with respect to $a_{i}$ and $b_{j}$, we construct the system $(i, j=1,2,3)$

$$
a_{i}=\frac{x_{i}+\sum_{j} T_{i j} b_{j}}{1+\sum_{j} b_{j}^{2}}, \quad b_{j}=\frac{y_{j}+\sum_{i} T_{i j} a_{i}}{1+\sum_{i} a_{i}^{2}} .
$$

In the case of $X$ states, defined in Eqs. (7) and (8), the above system reduces to

$$
\begin{aligned}
& a_{1}=\frac{T_{11} b_{1}+T_{12} b_{2}}{1+\sum_{j} b_{j}^{2}}, \quad b_{1}=\frac{T_{11} a_{1}+T_{21} a_{2}}{1+\sum_{j} a_{j}^{2}}, \\
& a_{2}=\frac{T_{21} b_{1}+T_{22} b_{2}}{1+\sum_{j} b_{j}^{2}}, \quad b_{2}=\frac{T_{12} a_{1}+T_{22} a_{2}}{1+\sum_{j} a_{j}^{2}}, \\
& a_{3}=\frac{x_{3}+T_{33} b_{3}}{1+\sum_{j} b_{j}^{2}}, \quad b_{3}=\frac{y_{3}+T_{33} a_{3}}{1+\sum_{i} a_{i}^{2}} .
\end{aligned}
$$


It can be shown (see Appendix A) that the absolute minimum of $F$ is obtained by putting $a_{1}=a_{2}=b_{1}=b_{2}=0$ and by taking the solutions for $a_{3}, b_{3}$ of the system

$$
a_{3}=\frac{x_{3}+T_{33} b_{3}}{1+b_{3}^{2}}, \quad b_{3}=\frac{y_{3}+T_{33} a_{3}}{1+a_{3}^{2}} .
$$

Indicating with $\bar{a}_{3}$ and $\bar{b}_{3}$ the solutions of Eqs. (13) and substituting in Eq. (9), the product state closest to an $X$ state has the form

$$
\pi_{\rho_{X}}=\frac{1}{4}\left[\mathbb{1} \otimes \mathbb{1}+\bar{a}_{3} \sigma_{3} \otimes \mathbb{1}+\bar{b}_{3} \mathbb{1} \otimes \sigma_{3}+\bar{a}_{3} \bar{b}_{3} \sigma_{3} \otimes \sigma_{3}\right]
$$

An important point, as we shall see later, is that the parameters $\bar{a}_{3}, \bar{b}_{3}$ only depend on $x_{3}, y_{3}, T_{33}$, while $T_{11}, T_{12}, T_{21}, T_{22}$ do not play any role.

We observe that, in general, $\bar{a}_{3} \neq x_{3}$ and $\bar{b}_{3} \neq y_{3}$, which means that the product state closest to an $X$ state in the square-norm distance measure is not given by the product of its marginals $\operatorname{Tr}_{B}\left(\rho_{X}\right) \otimes \operatorname{Tr}_{A}\left(\rho_{X}\right)$, in contrast to what happens for any quantum state when the distance is measured by the relative entropy [12].

\section{CLOSEST CLASSICAL STATE AND ITS CLOSEST PRODUCT STATE}

We now face the problem of finding explicit expressions of the classical state closest to a an $X$ state in the square-norm distance measure. For this purpose, we follow a reported procedure that permits us to obtain the closest classical state given an arbitrary two-qubit state [28]. We point out that, although explicit expressions for geometric quantum discord for $X$ states have been already reported in the literature [39], this is not the case for the expressions of the corresponding closest classical states. Here we also give the product state closest to the obtained closest classical state, which is required for calculating the geometric quantifier of classical correlations [see Eq. (4)].

The general result for geometric quantum discord of twoqubit states is $D_{\mathrm{g}}(\rho)=\frac{1}{4}\left[\|\vec{x}\|^{2}+\|T\|^{2}-k_{\max }\right]$, where $k_{\max }$ is the largest eigenvalue of the matrix $K=\vec{x} \vec{x}^{T}+T T^{T}\left(T^{T}\right.$ is the transpose of matrix $T$ ) [28]. The eigenvalues of matrix $K$ for an $X$ state, in terms of the density-matrix elements, are

$$
\begin{aligned}
& k_{1}=4\left(\rho_{14}+\rho_{23}\right)^{2}, \quad k_{2}=4\left(\rho_{14}-\rho_{23}\right)^{2}, \\
& k_{3}=2\left[\left(\rho_{11}-\rho_{33}\right)^{2}+\left(\rho_{22}-\rho_{44}\right)^{2}\right] .
\end{aligned}
$$

We observe that $k_{1}$ is always larger than $k_{2}$, so only two distinct cases have to be separately treated, that is, $k_{1} \leqslant k_{3}$ and $k_{1}>k_{3}$.

The closest classical state $\chi_{\rho}$ is obtainable through a minimization procedure with respect to the parameters $\vec{x}, \vec{y}, T$ of the original state $\rho$ expressed in the Bloch representation [28]. In the following we use this procedure to obtain, for these two cases, the explicit expressions of the closest classical state and of its closest product state for an $X$ state defined by the parameters of the Bloch representation given in Eq. (8).

\section{A. Case 1: $k_{1} \leqslant k_{3}$}

When the $X$ state has density-matrix elements such that the condition $k_{1} \leqslant k_{3}$ is fulfilled, we find that its closest classical state in the square-norm distance measure has the form

$$
\chi_{\rho_{X}}^{(1)}=\frac{1}{4}\left[\mathbb{1} \otimes \mathbb{1}+x_{3} \sigma_{3} \otimes \mathbb{1}+y_{3} \mathbb{1} \otimes \sigma_{3}+T_{33} \sigma_{3} \otimes \sigma_{3}\right],
$$

where the superscript (1) refers to case $1\left(k_{1} \leqslant k_{3}\right)$ of our analysis.

Seeing that $\chi_{\rho_{X}}^{(1)}$ is still an $X$ state, we can apply the results of Sec. IV to calculate its closest product state $\pi_{\chi_{\rho_{X}}}^{(1)}$. The diagonal elements of $\rho_{X}$ and $\chi_{\rho_{X}}$ are equal, $\left(\rho_{X}^{(1)}\right)_{i i}=\left(\chi_{\rho_{X}^{(1)}}\right)_{i i}$, and as said before, the solutions $\bar{a}_{3}$ and $\bar{b}_{3}$ of Eq. (13) only depend on the components $x_{3}, y_{3}, T_{33}$ containing the diagonal density-matrix elements. As a consequence, for $X$ states lying in case 1 , the product state closest to $\chi_{\rho_{X}}^{(1)}$ coincides with the product state closest to $\rho_{X}$ given in Eq. (14): $\pi_{\chi_{\rho_{X}}}^{(1)}=\pi_{\rho_{X}}$.

\section{B. Case 2: $k_{1}>k_{3}$}

If the $X$ state has density-matrix elements such that $k_{1}>k_{3}$, we obtain for the closest classical state

$$
\begin{aligned}
\chi_{\rho_{X}}^{(2)}= & \frac{1}{4}\left\{\mathbb{1} \otimes \mathbb{1}+y_{3} \mathbb{1} \otimes \sigma_{3}+\tilde{T}_{11} \sigma_{1} \otimes \sigma_{1}\right. \\
& \left.+\tilde{T}_{12} \sigma_{1} \otimes \sigma_{2}+\tilde{T}_{21} \sigma_{2} \otimes \sigma_{1}+\tilde{T}_{22} \sigma_{2} \otimes \sigma_{2}\right\},
\end{aligned}
$$

where the superscript (2) refers to the case $2\left(k_{1}>k_{3}\right)$ of our analysis and

$$
\begin{aligned}
\tilde{T}_{11} & =\frac{1}{2}\left\{T_{11}\left[1+\cos \left(\gamma_{14}+\gamma_{23}\right)\right]-T_{21} \sin \left(\gamma_{14}+\gamma_{23}\right)\right\} \\
& =\left[\cos \left(\gamma_{23}\right)+\cos \left(\gamma_{14}\right)\right]\left(\rho_{14}+\rho_{23}\right), \\
\tilde{T}_{12} & =\frac{1}{2}\left\{T_{12}\left[1+\cos \left(\gamma_{14}+\gamma_{23}\right)\right]-T_{22} \sin \left(\gamma_{14}+\gamma_{23}\right)\right\} \\
& =\left[\sin \left(\gamma_{23}\right)-\sin \left(\gamma_{14}\right)\right]\left(\rho_{14}+\rho_{23}\right), \\
\tilde{T}_{21} & =\frac{1}{2}\left\{T_{21}\left[1-\cos \left(\gamma_{14}+\gamma_{23}\right)\right]-T_{11} \sin \left(\gamma_{14}+\gamma_{23}\right)\right\} \\
& =-\left[\sin \left(\gamma_{23}\right)+\sin \left(\gamma_{14}\right)\right]\left(\rho_{14}+\rho_{23}\right), \\
\tilde{T}_{22} & =\frac{1}{2}\left\{T_{22}\left[1-\cos \left(\gamma_{14}+\gamma_{23}\right)\right]-T_{12} \sin \left(\gamma_{14}+\gamma_{23}\right)\right\} \\
& =\left[\cos \left(\gamma_{23}\right)-\cos \left(\gamma_{14}\right)\right]\left(\rho_{14}+\rho_{23}\right) .
\end{aligned}
$$

Here again $\chi_{\rho_{X}}^{(2)}$ has an $X$ structure, so considerations made in Sec. IV apply when one looks for its closest product state. In particular, solving Eq. (13) for $\chi_{\rho_{X}}^{(2)}$, the closest product state is found to be

$$
\pi_{\chi_{\rho_{X}}}^{(2)}=\frac{1}{4}\left[\mathbb{1} \otimes \mathbb{1}+y_{3} \mathbb{1} \otimes \sigma_{3}\right]
$$

For an $X$ state belonging to case 2 it results, differently from case 1 , that the closest product state to $\rho_{X}$ is different from the product state closest to $\chi_{\rho_{X}}^{(2)}: \pi_{\chi_{\rho_{X}}^{(2)}} \neq \pi_{\rho_{X}}$.

\section{GEOMETRIC CORRELATION QUANTIFIERS AND THEIR RELATIONS}

After obtaining the relevant closest states to an $X$ state, we are now able to give the explicit expressions of the geometric quantifiers of the various kinds of correlations, $T_{\mathrm{g}}, D_{\mathrm{g}}$, and $C_{\mathrm{g}}$, and to investigate their relations. 
From the findings of Sec. IV on the closest product state, it follows that the geometric quantifier of total correlations defined in Eq. (4) for an $X$ state is

$$
\begin{aligned}
T_{\mathrm{g}}\left(\rho_{X}\right)= & \frac{\left(x_{3}-\bar{a}_{3}\right)^{2}+\left(y_{3}-\bar{b}_{3}\right)^{2}+\left(T_{33}-\bar{a}_{3} \bar{b}_{3}\right)^{2}}{4} \\
& +\frac{1}{4}\left(T_{11}^{2}+T_{12}^{2}+T_{21}^{2}+T_{22}^{2}\right)
\end{aligned}
$$

for both cases 1 and 2 . We point out that, in general, $\operatorname{Tr}\left(\rho \pi_{\rho}\right) \neq$ $\operatorname{Tr}\left(\pi_{\rho}^{2}\right)$, and therefore $T_{\mathrm{g}}\left(\rho_{X}\right) \neq \operatorname{Tr}\left(\rho_{X}^{2}\right)-\operatorname{Tr}\left(\pi_{\rho_{X}}^{2}\right)$. Thus, total correlations measured by the square-norm distance are not expressible as the difference between purities, and they are not suitable to be represented by an arrow from a state to another, which happens for REB correlation quantifiers.

Concerning the geometric quantifiers of quantum and classical correlations, $D_{\mathrm{g}}$ and $C_{\mathrm{g}}$, respectively, we can obtain their explicit expressions from the results of Sec. V on the closest classical state and its closest product state, distinguishing the two cases $k_{1} \leqslant k_{3}$ (case 1$)$ and $k_{1}>k_{3}$ (case 2 ).

For $X$ states belonging to case $1\left(k_{1} \leqslant k_{3}\right)$, the geometric quantifiers of quantum and classical correlations defined in Eqs. (3) and (4) are

$$
\begin{aligned}
& D_{\mathrm{g}}^{(1)}=\frac{1}{4}\left(T_{11}^{2}+T_{12}^{2}+T_{21}^{2}+T_{22}^{2}\right)=2\left(\rho_{14}^{2}+\rho_{23}^{2}\right), \\
& C_{\mathrm{g}}^{(1)}=\frac{\left(x_{3}-\bar{a}_{3}\right)^{2}+\left(y_{3}-\bar{b}_{3}\right)^{2}+\left(T_{33}-\bar{a}_{3} \bar{b}_{3}\right)^{2}}{4} .
\end{aligned}
$$

Analogously to $T_{\mathrm{g}}, C_{\mathrm{g}}=\left\|\chi_{\rho}-\pi_{\chi_{\rho}}\right\|^{2}$ is not expressible as the difference between the purities of the states $\chi_{\rho}$ and $\pi_{\chi_{\rho}}$, and it is not representable in the same spirit of Eq. (2) by an arrow. Regarding the quantity $L_{\mathrm{g}}$ defined in Eq. (5), we know from Sec. V that, in case $1, \pi_{\chi_{\rho_{X}}}^{(1)}=\pi_{\rho_{X}}^{(1)}$ so that $L_{g}^{(1)}=0$. From Eqs. (20) and (21) we then observe that, in this case, among the geometric quantifiers of correlations the additivity relation

$$
T_{\mathrm{g}}=D_{\mathrm{g}}^{(1)}+C_{\mathrm{g}}^{(1)}
$$

holds, analogous to the one in Eq. (1) for REB correlation quantifiers with $L=0$.

On the other hand, for $X$ states belonging to case $2\left(k_{1}>k_{3}\right)$ the geometric quantifiers of quantum and classical correlations turn out to be

$$
\begin{aligned}
& D_{\mathrm{g}}^{(2)}=\left(\rho_{14}-\rho_{23}\right)^{2}+\frac{1}{2}\left[\left(\rho_{11}-\rho_{33}\right)^{2}+\left(\rho_{22}-\rho_{44}\right)^{2}\right], \\
& C_{\mathrm{g}}^{(2)}=\left(\rho_{14}+\rho_{23}\right)^{2},
\end{aligned}
$$

where the expressions are given in terms of the density-matrix elements because they are simpler than those in terms of the Bloch parameters. From Eqs. (20) and (23) we immediately notice that, in this case, an additivity relation analogous to that of Eq. (1) does not occur. In fact, we have

$$
T_{\mathrm{g}}-D_{\mathrm{g}}^{(2)}-C_{\mathrm{g}}^{(2)}=\bar{a}_{3}^{2} \frac{\left(T_{33}-\bar{a}_{3} \bar{b}_{3}\right)^{2}-\left(1+\bar{b}_{3}^{2}\right)}{4} .
$$

It can be shown (the proof is given in Appendix B) that, in general, $T_{\mathrm{g}}-D_{\mathrm{g}}^{(2)}-C_{\mathrm{g}}^{(2)} \leqslant 0$, and the equality holds if and only if $x_{3}+y_{3} T_{33}=0$. In order to estimate the typical value of $T_{\mathrm{g}}-D_{\mathrm{g}}^{(2)}-C_{\mathrm{g}}^{(2)}$, we have generated $10^{4} \mathrm{X}$-shaped random density matrices belonging to the case $k_{1}>k_{3}$, from which we have then numerically obtained $\bar{a}_{3}$ and $\bar{b}_{3}$ as solutions of

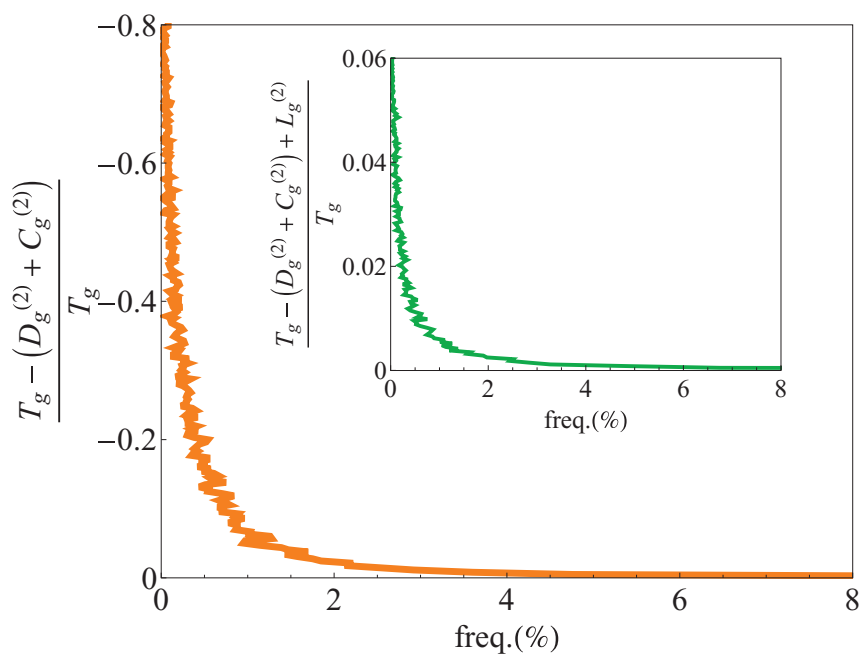

FIG. 2. (Color online) Values of the relative difference $\left(T_{\mathrm{g}}-\right.$ $\left.D_{\mathrm{g}}^{(2)}-C_{\mathrm{g}}^{(2)}\right) / T_{\mathrm{g}}$ of the geometric correlation quantifiers as a function of the probability of their occurrence for a two-qubit $X$ state belonging to case $2\left(k_{1}>k_{3}\right)$. The inset shows the values of $\left(T_{\mathrm{g}}+L_{\mathrm{g}}^{(2)}-D_{\mathrm{g}}^{(2)}-C_{\mathrm{g}}^{(2)}\right) / T_{\mathrm{g}}$ as a function of the probability of their occurrence for a two-qubit $X$ state also belonging to case 2. Some $10^{4}$ random density matrices have been produced.

Eq. (13) and finally calculated all the correlations, Eqs. (20) and (23). In Fig. 2, we plot the values of $\left(T_{\mathrm{g}}-D_{\mathrm{g}}^{(2)}-C_{\mathrm{g}}^{(2)}\right) / T_{\mathrm{g}}$ versus the (unnormalized) probability of having those amounts for an $X$ state for which $k_{1}>k_{3}$ (case 2). It is possible to see that there exist a nonnegligible number of states for which this difference is significantly different from zero.

In analogy to what happens for REB correlations in Eq. (1), one may, however, wonder if the quantity $L_{\mathrm{g}}$ of Eq. (5) can be used to close the loop of geometric correlations. We first observe that, as we know from Sec. V, in case $2 \pi_{\rho_{X}} \neq \pi_{\chi_{\rho_{X}}^{(2)}}$ and then $L_{\mathrm{g}}^{(2)} \neq 0$. In particular, it is

$$
L_{\mathrm{g}}^{(2)}=\bar{a}_{3}^{2} \frac{\left(T_{33}-\bar{a}_{3} \bar{b}_{3}\right)^{2}+\left(1+\bar{b}_{3}^{2}\right)}{4},
$$

so that

$$
T_{\mathrm{g}}-D_{\mathrm{g}}^{(2)}-C_{\mathrm{g}}^{(2)}+L_{\mathrm{g}}^{(2)}=\frac{\bar{a}_{3}^{2}}{2}\left(T_{33}-\bar{a}_{3} \bar{b}_{3}\right)^{2} .
$$

As a consequence, unless $\bar{a}_{3}=0$, which would also imply $L_{\mathrm{g}}^{(2)}=0$, it is impossible to have a closed additive relation among the different kinds of correlations when measured by the square norm. In other words, geometric quantifiers of correlations can be cast in a closed additivity relation only for $X$ states with $L_{\mathrm{g}}$ equal to zero. To quantitatively investigate this aspect, in the inset of Fig. 2, for the same $10^{4}$ randomly generated states as before, we plot values of $\left(T_{\mathrm{g}}+L_{\mathrm{g}}^{(2)}-D_{\mathrm{g}}^{(2)}-C_{\mathrm{g}}^{(2)}\right) / T_{\mathrm{g}}$ as a function of the probability of their occurrence for a two-qubit $X$ state also belonging to case 2. Even if the violation is, in general, small, it is possible to find states for which it is meaningful. An important subclass of the $X$ states is given by the Bell diagonal states, for which a closed additivity relation holds among the correlation quantifiers, as discussed in Appendix C. 
We here point out that the numerical results above are useful for further highlighting the qualitative conceptual aspect on the impossibility of finding, in general, a closed relation among the geometric correlation quantifiers. For example, there could be states, outside the class of $X$ states, for which the differences $T_{\mathrm{g}}-D_{\mathrm{g}}^{(2)}-C_{\mathrm{g}}^{(2)}$ and $T_{\mathrm{g}}+L_{\mathrm{g}}^{(2)}-D_{\mathrm{g}}^{(2)}-C_{\mathrm{g}}^{(2)}$ are larger than those we numerically find here for $X$ states. Moreover, as discussed before, another critical point arising when using the square-norm distance measure to quantify correlations is the absence of a "direction" in the correlation measures. While REB correlation quantifiers can be represented by arrows with precise directions from one state to another one, as Eqs. (2) and Fig. 1 clearly indicate, this is no longer true when geometric quantifiers of correlations are taken into account.

\section{PHYSICAL MODEL}

In this section we consider a specific physical model where the two state space zones $k_{1} \leqslant k_{3}$ and $k_{1}>k_{3}$ investigated above can be dynamically connected. In particular, we take two noninteracting qubits, $A$ and $B$, embedded in separated cavities and subject to a non-Markovian dynamics, as already reported in Ref. [40]. Each qubit interacts only and independently with its local environment, so that the total Hamiltonian is $H_{\text {tot }}=$ $H_{A}+H_{B}$. The single "qubit + reservoir" Hamiltonian $H_{S}$ $(S=A, B)$ is given by $(\hbar=1)$

$$
H_{S}=\omega_{0}^{S} \sigma_{+}^{S} \sigma_{-}^{S}+\sum_{k} \omega_{k} b_{k}^{S \dagger} b_{k}^{S}+\sum_{k}\left(g_{k}^{S} \sigma_{+}^{S} b_{k}^{S}+g_{k}^{S *} \sigma_{-}^{S} b_{k}^{S \dagger}\right) \text {, }
$$

where $\omega_{0}^{S}$ is the transition frequency of the two-level system (qubit) $S, \sigma_{ \pm}^{S}$ are the system raising and lowering operators, the index $k$ labels the field modes of the reservoir $S$ with frequencies $\omega_{k}, b_{k}^{S \dagger}$ and $b_{k}^{S}$ are the mode creation and annihilation operators, and $g_{k}^{S}$ are the coupling constants. We will consider the case where both Hamiltonians $H_{A}$ and $H_{B}$ have the same parameters. The Hamiltonian of Eq. (27) may represent a qubit made by the excited and ground electronic states of a two-level atom interacting with a reservoir given by the quantized modes of a high- $Q$ cavity [41], and it can also be implemented by superconducting Josephson qubits in the framework of circuit QED [42] and by entangled polarization photons in an all-optical setup [43]. If the two-qubit state has initially an $X$ structure, this is maintained during the dynamics locally governed by the Hamiltonian of Eq. (27), and the density-matrix elements at time $t$, in the same basis as in Eq. (7), are [40]

$$
\begin{aligned}
\rho_{11}(t) & =\rho_{11}(0) P_{t}^{2}, \\
\rho_{22}(t) & =\rho_{22}(0) P_{t}+\rho_{11}(0) P_{t}\left(1-P_{t}\right), \\
\rho_{33}(t) & =\rho_{33}(0) P_{t}+\rho_{11} P_{t}\left(1-P_{t}\right), \\
\rho_{44}(t) & =1-\left[\rho_{11}(t)+\rho_{22}(t)+\rho_{33}(t)\right], \\
\left|\rho_{14}(t)\right| & =\left|\rho_{14}(0)\right| P_{t}, \quad\left|\rho_{23}(t)\right|=\left|\rho_{23}(0)\right| P_{t},
\end{aligned}
$$

where $\quad P_{t}=e^{-\lambda t}\left[\cos \left(\frac{d t}{2}\right)+\frac{\lambda}{d} \sin \left(\frac{d t}{2}\right)\right]^{2}, \quad$ with $\quad d=$ $\sqrt{2 \gamma_{0} \lambda-\lambda^{2}}$. The parameter $\lambda$ represents the spectral width of the coupling, while $\gamma_{0}$ is the spontaneous emission rate of the qubit (atom). Under this evolution the two-qubit states may cross the two zones, $k_{1} \leqslant k_{3}$ and $k_{1}>k_{3}$, of Sec. V. This can be explicitly seen by choosing, for example,

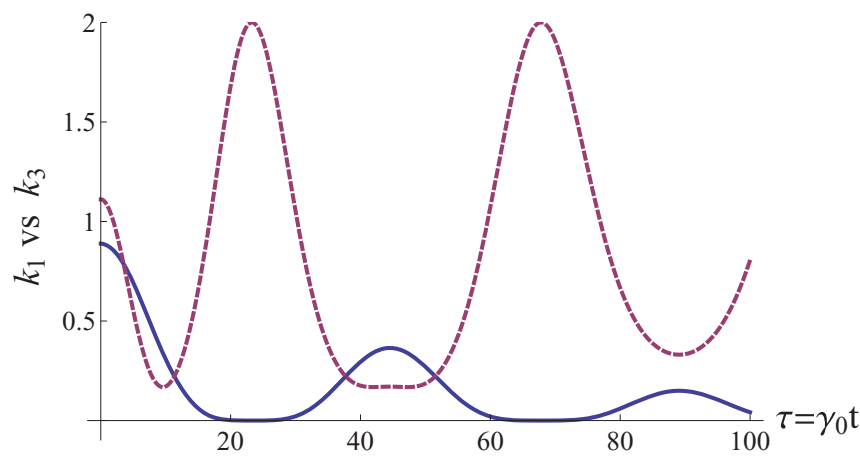

FIG. 3. (Color online) $k_{1}$ (blue solid line) vs $k_{3}$ (purple dashed line) as a function of the dimensionless time $\gamma_{0} t$ for $\lambda=0.01 \gamma_{0}$ and starting from the state $|\Psi\rangle=\sqrt{1 / 3}|00\rangle+\sqrt{2 / 3}|11\rangle$.

the initial state $|\Psi\rangle=\sqrt{1 / 3}|00\rangle+\sqrt{2 / 3}|11\rangle$ and $\lambda=0.01 \gamma_{0}$ (strong-coupling regime). In Fig. 3 we plot $k_{1}$ and $k_{3}$ as functions of the dimensionless time $\gamma_{0} t$. The plot clearly displays that there are time regions when $k_{1}$ is larger than $k_{3}$ and vice versa.

\section{CONCLUSIONS}

The distance between a quantum state and its closest states without certain properties has been employed in the literature to quantify in a unified view the various kinds of correlations present in an arbitrary multipartite quantum system. Using relative entropy as a distance measure, an additivity relation among total, $T$, quantum, $D$, and classical, $C$, correlations holds [12]. This additivity relation is of the kind $T=D+C-$ $L$ and contains a quantity $L$ defined as the relative entropybased distance between two particular closest product states linked to the system state [12].

In this paper we have investigated how a unified view of various kinds of correlations works in terms of a different distance measure. Among the possible suitable distance measures, we have considered the square-norm (Hilbert-Schmidt) distance, inspired by the fact that it has been already exploited to define geometric quantum discord $D_{\mathrm{g}}$ to quantify quantum correlations present in a state [28]. Using the square norm, we have then considered the geometric quantifiers for classical, $C_{\mathrm{g}}$, and total, $T_{\mathrm{g}}$, correlations, which have recently been introduced [32], and defined a quantity $L_{\mathrm{g}}$, analogous to the REB quantity $L$. We have given explicit expressions of the geometric correlation quantifiers for the class of two-qubit $X$ states $\rho_{X}$ by first finding the relevant closest states linked to $\rho_{X}$. We have then analyzed the relationships among the various correlation quantifiers, and we have shown that there exists a subclass of $X$ states for which it is not possible to find a closed additivity relation of the kind $T_{\mathrm{g}}=D_{\mathrm{g}}+C_{\mathrm{g}}-L_{\mathrm{g}}$. The additivity relation holding when relative entropy is used as distance measure is therefore not preserved when one quantifies in a unified way the different kinds of correlations present in a quantum state by using the square norm. Moreover we have explored numerically the abundance and importance of the nonadditivity of geometric correlations and found that there is a nonnegligible amount of $X$ states with a meaningful deviation from additivity. Therefore, both the analytical proof given for a subclass of $X$ states and the numerically evaluated 
occurrence among $X$ states show that, for a two-qubit random state, a closed relation among geometric correlation quantifiers does not need to be satisfied.

The results of this paper seem to confirm that different distance measures cannot serve equally well to quantify the various kinds of correlations present in a quantum state. In this sense, in Ref. [32] it had been shown that, when one compares the dynamics of correlation quantifiers based on relative entropy with that of correlation quantifiers based on the square-norm distance, different qualitative behaviors may occur. These findings recall the known result in the entanglement theory that different entanglement measures may induce different orderings in the state space [44]. The present results give a clear indication that different distance measures (in particular relative entropy and the square norm) may not share important general qualitative properties, such as the occurrence of closed additivity relations among the various correlation quantifiers.

\section{APPENDIX A}

In this appendix we show that the function $F$ of Eq. (10), measuring the distance between a two-qubit state $\rho$ and the arbitrary product state $\rho_{A} \otimes \rho_{B}$ of Eq. (9), has an absolute minimum for $a_{1}=a_{2}=b_{1}=b_{2}=0$ and for $a_{3}=\bar{a}_{3}, b_{3}=\bar{b}_{3}$ solutions of Eqs. (13) when an $X$ state is considered.

Let us consider the function $F$ of Eq. (10) in the case of $X$ states. It can be divided into two parts. We write $F=F_{1}+F_{2}$, where

$$
\begin{aligned}
F_{1}= & \frac{1}{4}\left[a_{1}^{2}+b_{1}^{2}+a_{2}^{2}+b_{2}^{2}+\left(T_{11}-a_{1} b_{1}\right)^{2}+\left(T_{12}-a_{1} b_{2}\right)^{2}\right. \\
& +\left(T_{21}-a_{2} b_{1}\right)^{2}+\left(T_{22}-a_{2} b_{1}\right)^{2} \\
& \left.+a_{1}^{2} b_{3}^{2}+a_{2}^{2} b_{3}^{2}+a_{3}^{2} b_{1}^{2}+a_{3}^{2} b_{2}^{2}\right]
\end{aligned}
$$

and

$$
F_{2}=\frac{1}{4}\left[\left(x_{3}-a_{3}\right)^{2}+\left(y_{3}-b_{3}\right)^{3}+\left(T_{33}-a_{3} b_{3}\right)^{2}\right] .
$$

We observe that $F_{2}$ only depends on $a_{3}$ and $b_{3}$ and also that, if $a_{1}=b_{1}=a_{2}=b_{2}=0$ gives a minimum for $F_{1}$, this occurs irrespective of the values of $a_{3}$ and $b_{3}$. Therefore, the absolute minimum of $F$ is obtained for the values of variables giving the absolute minimum of $F_{1}$ and $F_{2}$ separately. The absolute minimum of the convex function $F_{2}$ is just obtained in the values $a_{3}=\bar{a}_{3}$ and $b_{3}=\bar{b}_{3}$, which are solutions of Eqs. (13). In order to verify that $F_{1}$ actually has a minimum in $a_{1}=b_{1}=$ $a_{2}=b_{2}=0$, it is enough to consider the part of function $F_{1}$ not including $a_{3}$ and $b_{3}$ (the remaining part is equal to zero for $a_{1}=b_{1}=a_{2}=b_{2}=0$ ). Thus we focus on the function $f=f\left(a_{1}, b_{1}, a_{2}, b_{2}\right)$ given by

$$
\begin{aligned}
f= & a_{1}^{2}+b_{1}^{2}+a_{2}^{2}+b_{2}^{2}+\left(T_{11}-a_{1} b_{1}\right)^{2}+\left(T_{12}-a_{1} b_{2}\right)^{2} \\
& +\left(T_{21}-a_{2} b_{1}\right)^{2}+\left(T_{22}-a_{2} b_{1}\right)^{2} .
\end{aligned}
$$

In order to show that $f$ has an absolute minimum in $a_{1}=b_{1}=a_{2}=b_{2}=0$, we consider the difference $\Delta f=$ $f\left(a_{1}, b_{1}, a_{2}, b_{2}\right)-f(0,0,0,0)$ between the value of $f$ at any possible point and its value at $\{0,0,0,0\}$, that is,

$$
\begin{aligned}
\Delta f= & a_{1}^{2}+b_{1}^{2}+a_{2}^{2}+b_{2}^{2}+a_{1}^{2} b_{1}^{2}+a_{2}^{2} b_{2}^{2}+a_{2}^{2} b_{1}^{2}+a_{1}^{2} b_{2}^{2} \\
& -\left(T_{11} a_{1} b_{1}+T_{12} a_{1} b_{2}+T_{21} a_{2} b_{1}+T_{22} a_{2} b_{2}\right) .
\end{aligned}
$$

The last term of Eq. (A4) admits as a minimum value $-\left(\left|T_{11} a_{1} b_{1}\right|+\left|T_{12} a_{1} b_{2}\right|+\left|T_{21} a_{2} b_{1}\right|+\left|T_{22} a_{2} b_{2}\right|\right)$. Furthermore, since $\left|T_{i j}\right| \leqslant 1$, the lower bound of this expression is $-\left(\left|a_{1} b_{1}\right|+\left|a_{1} b_{2}\right|+\left|a_{2} b_{1}\right|+\left|a_{2} b_{2}\right|\right)$, so that

$$
\begin{aligned}
\Delta f \geqslant & a_{1}^{2}+b_{1}^{2}+a_{2}^{2}+b_{2}^{2}+a_{1}^{2} b_{1}^{2}+a_{2}^{2} b_{2}^{2}+a_{2}^{2} b_{1}^{2}+a_{1}^{2} b_{2}^{2} \\
& -\left(\left|a_{1} b_{1}\right|+\left|a_{1} b_{2}\right|+\left|a_{2} b_{1}\right|+\left|a_{2} b_{2}\right|\right) .
\end{aligned}
$$

We now observe that

$$
\begin{aligned}
a_{1}^{2}+b_{1}^{2}+a_{2}^{2}+b_{2}^{2}= & \left(\left|a_{1}\right|-\left|b_{1}\right|\right)^{2}+\left(\left|a_{2}\right|-\left|b_{2}\right|\right)^{2} \\
& +2\left|a_{1} b_{1}\right|+2\left|a_{2} b_{2}\right|
\end{aligned}
$$

and also

$$
\begin{aligned}
a_{1}^{2}+b_{1}^{2}+a_{2}^{2}+b_{2}^{2}= & \left(\left|a_{1}\right|-\left|b_{2}\right|\right)^{2}+\left(\left|a_{2}\right|-\left|b_{1}\right|\right)^{2} \\
& +2\left|a_{1} b_{2}\right|+2\left|a_{2} b_{1}\right| .
\end{aligned}
$$

Putting these expressions into Eq. (A5), we obtain

$$
\begin{aligned}
\Delta f \geqslant & \frac{1}{2}\left[\left(\left|a_{1}\right|-\left|b_{1}\right|\right)^{2}+\left(\left|a_{2}\right|-\left|b_{2}\right|\right)^{2}+\left(\left|a_{1}\right|-\left|b_{2}\right|\right)^{2}\right. \\
& \left.+\left(\left|a_{2}\right|-\left|b_{1}\right|\right)^{2}\right]+a_{1}^{2} b_{1}^{2}+a_{2}^{2} b_{2}^{2}+a_{2}^{2} b_{1}^{2}+a_{1}^{2} b_{2}^{2} .
\end{aligned}
$$

Therefore $\Delta f$ is always greater than zero unless $a_{1}=b_{1}=$ $a_{2}=b_{2}=0$.

\section{APPENDIX B}

In this appendix we want to prove that the quantity $h=$ $\bar{a}_{3}^{2}\left[\left(T_{33}-\bar{a}_{3} \bar{b}_{3}\right)^{2}-\left(1+\bar{b}_{3}^{2}\right)\right]$, where $\bar{a}_{3}, \bar{b}_{3}$ are solutions of Eqs. (13), which quantifies the difference $T_{\mathrm{g}}-D_{\mathrm{g}}^{(2)}-C_{\mathrm{g}}^{(2)}=$ $h / 4$ given in Eq. (24), satisfies the inequality $h \leqslant 0$ with the equality verified if and only if $x_{3}+y_{3} T_{33}=0$. To this aim, since $1+\bar{b}_{3}^{2} \geqslant 1$, it will be sufficient to prove that $\mid T_{33}-$ $\bar{a}_{3} \bar{b}_{3} \mid \leqslant 1$ and successively to find the condition for which the upper bound of 1 is achieved.

Let us start by noticing that $\bar{a}_{3}, \bar{b}_{3}$ give the minimum of the function $F_{2}$ of Eq. (A2). Thus, $F_{2}\left(a_{3}, b_{3}\right) \geqslant F_{2}\left(\bar{a}_{3}, \bar{b}_{3}\right) \geqslant$ $\left(T_{33}-\bar{a}_{3} \bar{b}_{3}\right)^{2}$. Then, if in Eq. (A2) we replace $a_{3}, b_{3}$ with $x_{3}, y_{3}$, the inequality

$$
\left(T_{33}-x_{3} y_{3}\right)^{2} \geqslant\left(T_{33}-\bar{a}_{3} \bar{b}_{3}\right)^{2}
$$

holds. Using Eqs. (8) of an $X$ state, we have

$$
T_{33}-x_{3} y_{3}=p_{1}-t_{1}^{2}-\left(p_{2}-t_{2}^{2}\right),
$$

where $p_{1}=\rho_{11}+\rho_{44}, p_{2}=1-p_{1}=\rho_{22}+\rho_{33}, t_{1}=\rho_{11}-$ $\rho_{44}$, and $t_{2}=\rho_{22}-\rho_{33} . T_{33}-x_{3} y_{3}$ reaches its maximum value for $t_{1}=0$ and $t_{2}=p_{2}$, while its minimum value is found for $t_{1}=p_{1}$ and $t_{2}=0$. In any case $\left(T_{33}-x_{3} y_{3}\right)_{\max }=p_{1}+p_{2}^{2}-$ $p_{2} \leqslant 1-p_{2}$ and $\left(T_{33}-x_{3} y_{3}\right)_{\min }=p_{1}-p_{1}^{2}-p_{2} \geqslant-1+$ $p_{1}$. Then $\left|T_{33}-x_{3} y_{3}\right| \leqslant 1$ and, according to inequality (B1), $\left|T_{33}-\bar{a}_{3} \bar{b}_{3}\right| \leqslant 1$, as we wanted to prove.

The cases such that $h=\bar{a}_{3}^{2}\left[\left(T_{33}-\bar{a}_{3} \bar{b}_{3}\right)^{2}-\left(1+\bar{b}_{3}^{2}\right)\right]=0$ correspond to (i) $\bar{a}_{3}=0$ or to (ii) $\bar{b}_{3}=0$ and $\left|T_{33}\right|=1$. From Eqs. (13), we can notice that case (i) implies $b_{3}=y_{3}$ and then $x_{3}+y_{3} T_{33}=0$; case (ii) instead implies that $\bar{a}_{3}=x_{3}= \pm y_{3}$, where $\pm y_{3}$ correspond to $T_{33}=\mp 1$, respectively, so that again $x_{3}+y_{3} T_{33}=0$.

\section{APPENDIX C}

In this appendix we discuss the properties of a particular subclass of $X$ states, namely, the Bell diagonal states, also 
called states with maximally mixed marginals [24]. Looking at the matrix form of an $X$ state given in Eq. (7), Bell diagonal states have diagonal elements $\rho_{11}=\rho_{44}, \rho_{22}=\rho_{33}$ and nondiagonal density-matrix elements that are real, that is, $\gamma_{14}, \gamma_{23}=0, \pi$. Therefore, the parameters of their Bloch representation are $x_{3}=y_{3}=T_{12}=T_{21}=0, T_{11}=2 e^{i \gamma_{14}}\left[\rho_{14}+\right.$ $\left.e^{i\left(\gamma_{14}-\gamma_{23}\right)} \rho_{23}\right], \quad T_{22}=2 e^{i \gamma_{14}}\left[e^{i\left(\gamma_{14}-\gamma_{23}\right)} \rho_{23}-\rho_{14}\right], \quad$ and $T_{33}=$ $2\left(\rho_{11}-\rho_{22}\right)$, giving a Bloch representation [24]:

$$
\rho^{\mathrm{B}}=\left[\mathbb{1} \otimes \mathbb{1}+\sum_{i=1}^{3} T_{i i} \sigma_{i} \otimes \sigma_{i}\right] / 4 .
$$

Bell diagonal states have the peculiar property that the quantity $L$ of Eq. (2) is zero, so that $T=D+C$ for REB correlation quantifiers [12]. Indeed, these states present the same closed additivity relation even for the geometric correlation quantifiers of Eqs. (3) and (4), that is, $T_{\mathrm{g}}=D_{\mathrm{g}}+C_{\mathrm{g}}$ [32], with $L_{\mathrm{g}}=0$.

Bell diagonal states $\rho^{\mathrm{B}}$ have $x_{3}=y_{3}=0$, and it is possible to show that the corresponding solutions of Eqs. (13) are $\bar{a}_{3}=$ $\bar{b}_{3}=0$ [32], so that the closest product state using the square norm, in this case, reduces to the product of the marginals $\pi_{\rho^{B}}=(\mathbb{1} / 2) \otimes(\mathbb{1} / 2)$.

Regarding the closest classical state and its closest product state (see Sec. V), for Bell diagonal states, if $\gamma_{14}=\gamma_{23}$, then $k_{i i}=T_{i i}^{2}$, while if $\gamma_{14}=\pi-\gamma_{23}$, one has $k_{11}=T_{22}^{2}, k_{22}=$ $T_{11}^{2}$ and always $k_{33}=T_{33}^{2}$. The two cases, $k_{1} \leqslant k_{3}$ and $k_{1}>$ $k_{3}$, obtained from Eq. (15) thus involve direct comparisons among absolute values of the components $T_{11}, T_{22}, T_{33}$ of the correlation tensor. Then, if a Bell diagonal state belongs to case $1\left(k_{1} \leqslant k_{3}\right)$, since $x_{3}=y_{3}=0$ the closest classical state of Eq. (16) reduces to $\chi_{\rho^{\mathrm{B}}}^{(1)}=\frac{1}{4}\left[\mathbb{1} \otimes \mathbb{1}+T_{33} \sigma_{3} \otimes \sigma_{3}\right]$, with $T_{33}^{2}=$ $k_{3}$. On the other hand, for a Bell diagonal state belonging to case $2\left(k_{1}>k_{3}\right)$, where $y_{3}=0$ and $\gamma_{14}, \gamma_{23}=0, \pi$, the closest classical state of Eq. (17) reduces either to $\chi_{\rho^{\mathrm{B}}}^{(2)}=\frac{1}{4}[\mathbb{1} \otimes \mathbb{1}+$ $\left.T_{11} \sigma_{1} \otimes \sigma_{1}\right]$ if $\gamma_{14}=\gamma_{23}$, with $T_{11}^{2}=k_{1}$, or to $\chi_{\rho^{\mathrm{B}}}^{(2)}=\frac{1}{4}[\mathbb{1} \otimes$ $\left.\mathbb{1}+T_{22} \sigma_{2} \otimes \sigma_{2}\right]$ if $\gamma_{14}=\pi-\gamma_{23}$, with $T_{22}^{2}=k_{1}$. Since we have already seen that the closest product state to $\rho^{\mathrm{B}}$ is given by the product of its marginals, the same happens for the product state closest to the closest classical state, $\chi_{\rho^{\mathrm{B}}}^{(1)}$ or $\chi_{\rho^{\mathrm{B}}}^{(2)}$.

For a Bell diagonal state the explicit expression of geometric quantum discord can be written as $D_{\mathrm{g}}\left(\rho^{\mathrm{B}}\right)=\frac{1}{4}\left[T_{11}^{2}+\right.$ $T_{22}^{2}+T_{33}^{2}-T^{2}$ ] [28], where $T \equiv \max \left\{\left|T_{11}\right|,\left|T_{22}\right|,\left|T_{33}\right|\right\}$. The geometric quantifier of total correlations of Eq. (20) reduces to $T_{\mathrm{g}}\left(\rho^{\mathrm{B}}\right)=\left(T_{11}^{2}+T_{22}^{2}+T_{33}^{2}\right) / 4$, with the geometric quantifier of classical correlations given by $C_{\mathrm{g}}\left(\rho^{\mathrm{B}}\right)=T^{2} / 4$ [32], so that it is always $T_{\mathrm{g}}=D_{\mathrm{g}}+C_{\mathrm{g}}$. In particular, for Bell diagonal states belonging to case 2, the right hand side of Eq. (24) is zero because $x_{3}+y_{3} T_{33}=0$, since $x_{3}=y_{3}=0$.
[1] J. S. Bell, Phys. Rev. 1, 195 (1964).

[2] A. Einstein, B. Podolsky, and N. Rosen, Phys. Rev. 47, 777 (1935).

[3] M. A. Nielsen and I. L. Chuang, Quantum Computation and Quantum Information (Cambridge University Press, Cambridge, 2000).

[4] R. Jozsa and N. Linden, Proc. R. Soc. A 459, 2011 (2003).

[5] E. Knill and R. Laflamme, Phys. Rev. Lett. 81, 5672 (1998); B. P. Lanyon, M. Barbieri, M. P. Almeida, and A. G. White, ibid. 101, 200501 (2008).

[6] A. Datta, A. Shaji, and C. M. Caves, Phys. Rev. Lett. 100, 050502 (2008).

[7] H. Ollivier and W. H. Zurek, Phys. Rev. Lett. 88, 017901 (2001).

[8] L. Henderson and V. Vedral, J. Phys. A 34, 6899 (2001).

[9] R. Auccaise, J. Maziero, L. C. Celeri, D. O. Soares-Pinto, E. R. de Azevedo, T. J. Bonagamba, R. S. Sarthour, I. S. Oliveira, and R. M. Serra, Phys. Rev. Lett. 107, 070501 (2011).

[10] G. Passante, O. Moussa, D. A. Trottier, and R. Laflamme, Phys. Rev. A 84, 044302 (2011).

[11] V. Vedral, M. B. Plenio, M. A. Rippin, and P. L. Knight, Phys. Rev. Lett. 78, 2275 (1997).

[12] K. Modi, T. Paterek, W. Son, V. Vedral, and M. Williamson, Phys. Rev. Lett. 104, 080501 (2010).

[13] L. C. Céleri, J. Maziero, and R. M. Serra, Int. J. Quant. Inf. 9, 1837 (2011).

[14] A. Ferraro, L. Aolita, D. Cavalcanti, F. M. Cucchietti, and A. Acín, Phys. Rev. A 81, 052318 (2010); F. Galve, G. L. Giorgi, and R. Zambrini, ibid. 83, 012102 (2011).

[15] M. F. Cornelio, M. C. de Oliveira, and F. F. Fanchini, Phys. Rev. Lett. 107, 020502 (2011).
[16] A. Streltsov, H. Kampermann, and D. Bruss, Phys. Rev. Lett. 106, 160401 (2011).

[17] M. Piani, S. Gharibian, G. Adesso, J. Calsamiglia, P. Horodecki, and A. Winter, Phys. Rev. Lett. 106, 220403 (2011).

[18] S. Campbell, T. J. G. Apollaro, C. Di Franco, L. Banchi, A. Cuccoli, R. Vaia, F. Plastina, and M. Paternostro, Phys. Rev. A 84, 052316 (2011); F. Ciccarello and V. Giovannetti, ibid. 85, 010102(R) (2012); A. Streltsov, H. Kampermann, and D. Bruss, Phys. Rev. Lett. 107, 170502 (2011); F. Ciccarello and V. Giovannetti, Phys. Rev. A 85, 022108 (2012).

[19] R. Prabhu, A. K. Pati, A. Sen De, and U. Sen, e-print arXiv:1108.5168; G. L. Giorgi, Phys. Rev. A 84, 054301 (2011); F. F. Fanchini, M. C. de Oliveira, L. K. Castelano, and M. F. Cornelio, e-print arXiv:1110.1054.

[20] T. Werlang, S. Souza, F. F. Fanchini, and C. J. Villas Boas, Phys. Rev. A 80, 024103 (2009).

[21] R. Lo Franco, E. Andersson, B. Bellomo, and G. Compagno, e-print arXiv:1009.5710.

[22] G. L. Giorgi, B. Bellomo, F. Galve, and R. Zambrini, Phys. Rev. Lett. 107, 190501 (2011); C. C. Rulli and M. S. Sarandy, Phys Rev. A 84, 042109 (2011); I. Chakrabarty, P. Agrawal, and A. K. Pati, Eur. Phys. J. D 65, 605 (2011).

[23] F. Galve, G. L. Giorgi, and R. Zambrini, Europhys. Lett. 96, 40005 (2011).

[24] S. Luo, Phys. Rev. A 77, 042303 (2008).

[25] L. X. Cen, X. Q. Li, J. Shao, and Y. J. Yan, Phys. Rev. A 83, 054101 (2011).

[26] M. Ali, A. R. P. Rau, and G. Alber, Phys. Rev. A 81, 042105 (2010).

[27] P. Giorda and M. G. A. Paris, Phys. Rev. Lett. 105, 020503 (2010); G. Adesso and A. Datta, ibid. 105, 030501 (2010). 
[28] B. Dakić, V. Vedral, and Č. Brukner, Phys. Rev. Lett. 105, 190502 (2010).

[29] J. Batle, A. Plastino, A. R. Plastino, and M. Casas, J. Phys. A 44, 505304 (2011).

[30] D. Girolami and G. Adesso, Phys. Rev. A 83, 052108 (2011).

[31] R. Prabhu, A. K. Pati, A. Sen De, and U. Sen, e-print arXiv:1109.4318.

[32] B. Bellomo, R. Lo Franco, and G. Compagno, e-print arXiv:1104.4043.

[33] S. Luo and S. Fu, Phys. Rev. A 82, 034302 (2010).

[34] B. Bellomo, R. Lo Franco, and G. Compagno, Adv. Sci. Lett. 2, 459 (2009).

[35] A. Chiuri, G. Vallone, M. Paternostro, and P. Mataloni, Phys. Rev. A 84, 020304(R) (2011); L. Di Carlo et al., Nature (London) 460, 240 (2009); N. A. Peters, J. B. Altepeter, D. A. Branning, E. R. Jeffrey, T.-C. Wei, and P. G. Kwiat, Phys. Rev. Lett. 92, 133601 (2004); J. S. Pratt, ibid. 93, 237205 (2004).
[36] T. J. Osborne and M. A. Nielsen, Phys. Rev. A 66, 032110 (2002); A. Osterloh, L. Amico, G. Falci, and R. Fazio, Nature (London) 416, 608 (2002).

[37] T. Yu and J. H. Eberly, Quantum Inf. Comput. 7, 459 (2007).

[38] A. R. P. Rau, J. Phys. A 42, 412002 (2009).

[39] F. Altintas, Opt. Commun. 283, 5264 (2010).

[40] B. Bellomo, R. Lo Franco, and G. Compagno, Phys. Rev. Lett. 99, 160502 (2007).

[41] S. Kuhr et al., Appl. Phys. Lett. 90, 164101 (2007).

[42] J. M. Fink, R. Bianchetti, M. Baur, M. Goppl, L. Steffen, S. Filipp, P. J. Leek, A. Blais, and A. Wallraff, Phys. Rev. Lett. 103, 083601 (2009).

[43] M. P. Almeida et al., Science 316, 579 (2007); J.-S. Xu, C. F. Li, M. Gong, X. B. Zou, C. H. Shi, G. Chen, and G. C. Guo, Phys. Rev. Lett. 104, 100502 (2010).

[44] J. Eisert and M. B. Plenio, J. Mod. Opt. 46, 145 (1999); S. Virmani and M. B. Plenio, Phys. Lett. A 268, 31 (2000); A. Miranowicz and A. Grudka, Phys. Rev. A 70, 032326 (2004). 